\title{
A pilot study of subjective well-being in colorectal cancer patients and their caregivers
}

\author{
This article was published in the following Dove Press journal: \\ Patient Related Outcome Measures \\ 19 October 2017 \\ Number of times this article has been viewed
}

\author{
Janet Graham' \\ Pavlina Spiliopoulou' \\ Rob Arbuckle ${ }^{2}$ \\ Julie Ann Bridge ${ }^{3}$ \\ James Cassidy'
}

'Department of Medical Oncology, Beatson, West of Scotland Cancer Center, Glasgow, UK, ${ }^{2}$ Adelphi Values, Adelphi Mill Macclesfield, Cheshire, UK; ${ }^{3}$ Eli Lilly Pty Ltd., West Ryde,

NSW, Australia
Correspondence: Pavlina Spiliopoulou Beatson, West of Scotland Cancer Center, 1053 Great Western Road, GI2 OYN, Glasgow, UK

Email pavlina.spiliopoulou@nhs.net
Background: Traditional endpoints in oncology are based on measuring the tumor size and combining this with a time factor. Current studies with immunotherapy show that even when median survival is unaltered, a significant proportion of patients can achieve prolonged survival. Objective tumor response does not always mean "overall" improvement, especially if toxicity is harsh. Novel agents are significantly expensive, and it is therefore crucial to measure the impact on "quality" of life, in addition to "quantity".

Materials and methods: We studied the preferences and experiences of cancer patients and their caregivers, measuring subjective well-being (SWB) ratings, EQ5D descriptions and time trade-off preferences.

Results: We studied 99 patients and 88 caregivers. Life satisfaction ratings were similar between the two groups, but daily mood was significantly lower in caregivers $(P<0.1)$. Anxiety/depression affected SWB, while pain and mobility did not. Positive thoughts about health were associated with better daily moods in both groups, and stage IV cancer was associated with lower life satisfaction. Cancer in remission was associated with better daily moods, but, interestingly, not with patient life satisfaction. Patients with better daily mood and positive thoughts about family were less willing to "trade-off" life years.

Conclusion: Caregivers are as anxious or depressed as patients, and report similar levels of life satisfaction but lower daily mood. A focus on SWB could provide a valid assessment of treatment benefit. Given the interesting results of this pilot study, we suggest a larger study should be conducted, measuring SWB over time.

Keywords: subjective well-being, EQ5D, TTO, life satisfaction, day affect

\section{Introduction}

Traditional endpoints in oncology are mainly based on objective measurement of tumor size combined with a time factor. Both the oncology community and the regulatory authorities rely heavily upon these endpoints to judge the benefit of new therapies. However, time-dependent variables such as median survival can be difficult to explain to patients and they lack individualization. Despite the widely accepted opinion that patients will accept any amount of additional time at any cost, this is not always the case. For patients, and their caregivers, quality and quantity of additional time go hand-in-hand, and even a modest gain in overall survival accompanied by significant toxicity might not be considered acceptable.

The question then is how do we reliably measure benefit and symptoms? And what could we do to make clinical endpoints more meaningful to individual patients?

Increasingly, "softer" measures of efficacy, such as symptom scores and Quality of Life (QoL) questionnaires, are being integrated into drug development process. Maisey 
et al demonstrated that patients with a higher baseline QoL do better. ${ }^{1,2}$ Quinten et al published a meta-analysis of 30 European Organisation for Research and Treatment of Cancer (EORTC) studies which demonstrated that QoL measures were statistically much more reliable than Eastern Cooperative Oncology Group performance status. ${ }^{3}$ With regards to symptoms assessment, Vera-Badillo et al analyzed 164 trials and demonstrated that symptoms and toxicity are poorly reported. ${ }^{4}$ Clinicians often rely on Common Terminology Criteria (CTC) grading to report toxicity, and although CTC provides a common language among physicians, it is often based on arbitrary cut-offs. The National Cancer Institute and the US Food and Drug Administration are now considering assessing common symptoms such as fatigue, nausea and diarrhea, directly from the patient rather the clinician, in view of mounting evidence that this method results in better symptom reporting.

While publicly funded health care aims to improve patients' health, it also requires that, ideally, the benefits of treatments should be compared to one another using a common currency. The currency most often considered is the Quality-Adjusted Life Year (QALY). The Q in the QALY is calibrated on a scale between 0 (for dead) and 1 (for full health). QALY calculations rely on the ability to describe the most important experiences of health, and generic health state descriptive systems, such as the EQ5D and Six-Form 6-Dimension (SF6D), have been devised in order to allow individuals to describe their current health by answering a number of questions. ${ }^{5,6} \mathrm{QALY}$ s then require that those states are valued. The National Institute of Clinical Excellence favors using the hypothetical preferences of members of the general public, who are asked to consider sacrifices in terms of risk of death (standard gamble) or length of life (time trade-off [TTO]) for improvements in QoL. ${ }^{7}$ There are limitations in this approach; firstly, in describing health in terms of a fixed and deliberately simplified descriptive system, we may fail to capture what is important to people in terms of their health. Second, in valuing health in terms of hypothetical preferences, we may fail to adequately anticipate the real impact that different health states have on our lives. In continuing with the status quo, we, therefore, run the real risk of misallocating resources. Also, if one takes a real-life scenario then, for example, the person who has a traumatic amputation of a leg in an accident - as expected, the QoL declines very markedly at first, but then returns to near normal or even normal with time. So, the patient adapts and accommodates to his/her disability with time. It seems likely that cancer patients experience this same phenomenon of resetting their baseline as their disease experience unfolds.
Recently, an alternative approach which values health states according to their effects on reports of subjective wellbeing (SWB) has been proposed. SWB is a general term for how we think and feel about life. The approach requires us to ask people very simple questions about their life satisfaction (LS) or mood, to find out about their health and other potentially important determinants of SWB, and then to use regression analysis to determine the marginal effects (the hit on SWB) of the various determinants.

If we are to more accurately capture the benefits of cancer treatments and other forms of health care, we need to move away from EQ5D and toward a broader conception of health and well-being, and away from the hypothetical preferences of the general public and toward the real experiences of real patients and their families. This perspective does not require us to abandon the QALY approach, but it does require us to value the $\mathrm{Q}$ rather differently. A focus on SWB becomes further justified in light of the robust relationship between health and $\mathrm{SWB}^{8}$ and the positive causal relationship from SWB to health. ${ }^{9,10}$

So, if we know that correlation of radiologic findings and clinical benefit might be sometimes weak, the CTC grading has limitations, and that we could potentially obtain reliable and clinically meaningful data from our patients, then this raises the question, what about their nearest and dearest? Caregivers are often closely involved in day-to-day care, and at consultations, they will often prompt patients to recall troublesome symptoms.

With cancer becoming more of a continuous care issue due to increasing incidence rates, improved survival and a movement toward outpatient treatment, more responsibility overseeing patients' care has been transferred onto their caregivers. ${ }^{11}$ Many studies have shown significant adverse effects of being a caregiver, including depression, sleep and appetite disorders, withdrawal from social engagements ${ }^{12}$ and a reduction in time they have for themselves, family and friends. ${ }^{13}$ Thirty-seven percent of the intensive caregivers (over 20 hours per week) have mental health scores that are consistent with a clinical diagnosis of depression, which is around $10 \%$ higher than that of noncaregivers. ${ }^{14}$

In this study, we set out to measure the SWB score, along with collecting other more established standard QoL assessments in a group of colorectal patients and their caregivers. The aim was to draw clinician's attention in considering SWB as a measure of the impact of cancer on the lives of patients and, crucially and innovatively, on the lives of a close family member (henceforth "caregiver"). We undertook a pilot study 
of the experiences of patients and their caregivers. The six objectives of this study were to:

1. Elicit SWB ratings from cancer patients and their caregivers.

2. Elicit EQ5D descriptions and TTO preferences from the same respondents.

3. Compare the SWB of patients and caregivers.

4. Compare the weights attached to dimensions of the EQ5D estimated from the various reports of SWB with those estimated from TTO responses.

5. Explore the relationships between SWB and the background characteristics of the respondents (including the EQ5D and the "cancer status" of the patient).

6. Explore the determinants of TTO values, including the effect of SWB measures.

\section{Materials and methods}

The study was open to recruitment between March 2010 and December 2010 in a single tertiary cancer center. It was approved by the West of Scotland Research Ethics Committee.

The final sample consisted of 99 colorectal cancer patients and 88 caregivers. Given these sample sizes, we used $10 \%$ level of statistical significance throughout (i.e., $P<0.1)$ and performed multivariable regression analysis.

The patient was asked to decide upon a family member who cares for his/her on a daily basis, and this person was asked to complete a questionnaire as a "caregiver".

\section{Questionnaire and coding}

The questionnaire consisted of the following sections: 1) SWB questions, 2) TTO (patient only), 3) EQ5D, 4) EORTC QLQ-C30 (patient only), 5) caregiver QoL (caregiver only), and 6) background characteristics.

Sections (4) and (5) are very detailed measures, and we exclude them from our analysis because our relatively small samples size $(n<100)$ means that we lack the degrees of freedom to generate meaningful results from regression analysis. We do, however, have some relevant variables about the disease stage and progression from (6). In any event, our main focus is on the relationship between (1), (2) and (3), within and between patients and caregivers.

The SWB questions were based on the state of the art. ${ }^{15}$ The first question was an evaluation of overall LS: "How satisfied are you with your life overall? On a scale of 0 to 100,100 being completely satisfied and 0 so dissatisfied you may as well be dead". The second question tapped into the experience of mood: "Overall how did you feel yesterday" (for each of the adjectives)? Hopeful, sad, fearful, tired, happy, worried, satisfied (on a 0-6 scale from "not at all" to "very much"). Mean day affect (DA) was calculated as the mean response to the positive emotion questions minus the mean response to the negative emotion questions.

These "headline indicators" are the main focus of our analyses, but they may neglect the way in which our attention drifts between current activities and concerns about other things (such as cancer). Research in psychology suggests such "mind wanderings" are frequent, occurring in up to $30 \%$ of randomly sampled moments during an average day. ${ }^{16}$ When these mind wanderings repeatedly return to the same issues, they are labeled as intrusive thoughts and they often have a negative association with our experienced utility. ${ }^{17,18}$ Respondents were, therefore, asked separately whether they had thought about health and then family during the previous day and were provided with the following responses from which to choose: "not at all, a few times, many times and continually". When the response was not "not at all", patients were then asked how "happy" and "worried" they felt when thinking about family or health on a scale from 0 (not at all) to 6 (very much). Responses were then coded into four categories in order of decreasing desirability: 1) thinking about health/family many times or continually with happy $\geq$ worried; 2) thinking about health/family a few times with happy $\geq$ worried; 3 ) thinking about health/family a few times with happy < worried; and 4) thinking about health/family many times or continually with happy $<$ worried.

In the TTO, patients (only) were asked to choose between 10 years "with your current health problems" and varying amounts of time "without any health problems", ranging from 0 to 10 years in intervals of 6 months. The midpoint between where a respondent switches his/her preferences is taken as his/her point of indifference, and the TTO value so calculated. For example, if a respondent preferred 6 years without problems to 10 years with problems but preferred 10 years with problems to 5.5 years without problems, the TTO value $=[(0.6+0.55) / 2]=0.575$.

For the EQ5D, respondents were asked to describe their health in terms of five dimensions (mobility, self-care, usual activities, pain, and mood), each with three levels of severity ( 1 =broadly none, $2=$ some and 3 =extreme problems). Each state is defined by a five-number code, from 11111 to 33333 , and so there are $243\left(3^{5}\right)$ possible states.

The background variables contained information including disease stage, type and number of treatments, concomitant conditions, as well as the more "standard" variables relating 
to age, marital status and education. Figures S1 and S2 show all the variables and coding used in the regression models.

Before conducting the study, potential participants who met the general inclusion criteria as well as cancer patientspecific inclusion criteria were identified: 1) oncologists were required to confirm cancer diagnosis in the patient; and 2) caregivers would accompany them at their clinic visit and also be willing and able to complete the appropriate study questionnaires. Exclusion criteria were: 1) severe acute or chronic medical or psychiatric conditions; 2) insufficient ability to read and write; and 3) being unable or unwilling to complete the questionnaires.

All participants provided written consent by signing either the patient or caregiver consent form (Version 1.0, September 2009). The Informed Consent Form explained to participants that anonymized study data would be stored in a computer database and confidentiality maintained in accordance with UK data legislation. Patients were instructed to designate a family member or caregiver on whom the patient relied for practical and emotional support.

To ensure a diverse sample of participants, efforts were made to recruit: 1) at least $30 \%$ of patients who were male and at least $30 \%$ who were female; 2 ) at least $30 \%$ of patients aged under 60 years; 3 ) at least $30 \%$ of patients in stage $1-2$ of colorectal cancer and at least $30 \%$ of patients in stage 3-4 of colorectal cancer; and 4) a minimum of $20 \%$ of patients who had not completed any education (college or university) beyond secondary school.

\section{Results and discussion}

There was an approximately even split of male:female in the 99 respondents in the patient group and the majority were Caucasian (Table 1). Caregivers were predominantly female. The patient group was older on average than the caregivers, and a larger proportion of them were retired. In terms of education, the patient group had largely completed less than a university degree: data on education were not available for caregivers. Patients overwhelmingly had stage III and IV cancer and approximately one-third of patients were classified as being in remission.

In Table 2, looking at the EQ5D, patients reported more physical limitations than caregivers, but, interestingly, reports of anxiety/depression were quite similar. Overall, more caregivers reported no problems on any of the dimensions ( $40 \%$ compared to $16 \%$ of patients). The mean TTO score was 0.825 , with around half of the patients unwilling to trade-off more than a year. The LS scores for patients
Table I Background information

\begin{tabular}{|c|c|c|}
\hline & $\begin{array}{l}\text { Patients } \\
n=99, \%\end{array}$ & $\begin{array}{l}\text { Caregivers } \\
\mathrm{n}=\mathbf{8 8}, \%\end{array}$ \\
\hline \multicolumn{3}{|l|}{ Age, years (mean) } \\
\hline$\geq 60$ & 70 & 46 \\
\hline$<60$ & 30 & 54 \\
\hline \multicolumn{3}{|l|}{ Gender } \\
\hline Male & 52 & 33 \\
\hline Female & 48 & 77 \\
\hline \multicolumn{3}{|l|}{ Ethnicity } \\
\hline Caucasian & 97 & 97 \\
\hline Non-Caucasian & 3 & \\
\hline \multicolumn{3}{|l|}{ Employment } \\
\hline Full-time & 17 & 24 \\
\hline Part-time & 2 & 12 \\
\hline Unemployed & 2 & 11 \\
\hline Retired & 62 & 38 \\
\hline Other & 17 & 15 \\
\hline \multicolumn{3}{|l|}{ Education } \\
\hline University or higher & 20 & N/A \\
\hline Less that university & 80 & N/A \\
\hline \multicolumn{3}{|l|}{ Cancer stage } \\
\hline Stage 0 & 2 & \\
\hline Stage 1 & 0 & \\
\hline Stage 2 & 11 & \\
\hline Stage 3 & 30 & \\
\hline Stage 4 & 53 & \\
\hline \multicolumn{3}{|l|}{ Remission status } \\
\hline In remission & 34 & \\
\hline Not in remission & 66 & \\
\hline \multicolumn{3}{|l|}{ Performance status } \\
\hline Fully active & 39 & \\
\hline Not fully active & 61 & \\
\hline \multicolumn{3}{|l|}{ Year of diagnosis } \\
\hline 2010 & 39 & \\
\hline Pre-20I0 & 61 & \\
\hline
\end{tabular}

Abbreviation: N/A, not available.

and caregivers were nearly identical at 78.74 and 78.48 , respectively, but patients had significantly higher DA: 2.36 and 1.97 , respectively.

Table 3 shows the results of patient/caregiver comparisons after controlling for the other variables that could significantly explain variation in LS and DA. There was no significant difference between patients and caregivers in LS, but caregivers had about a $24 \%$ lower DA score.

Table 4 shows the thoughts of patients and caregivers about health. Patients reported thinking more frequently and worrisomely about health than the caregiver group, while caregivers reported thinking more frequently and worrisomely about family than the patient group.

\section{Comparing the EQ5D scores}

Regression analysis was used to determine the impact of the EQ5D on SWB and TTO, in a similar way to our previous 
Table 2 EQ5D questionnaire responses for patients and caregivers

\begin{tabular}{|c|c|c|c|c|c|}
\hline Answers & Mobility, \% & Self-care, \% & Usual activities, \% & Pain/distress, \% & Anxiety/depression, \% \\
\hline \multicolumn{6}{|c|}{ EQ5D patients } \\
\hline Level I & 48 & 84 & 43 & 40 & 54 \\
\hline Level 2 & 52 & 14 & 50 & 51 & 41 \\
\hline Level 3 & 0 & 2 & 7 & 9 & 5 \\
\hline 11111 & & & 16 & & \\
\hline \multicolumn{6}{|c|}{ EQ5D carers } \\
\hline Level I & 87 & 94 & 85 & 63 & 55 \\
\hline Level 2 & 17 & 6 & 15 & 31 & 41 \\
\hline Level 3 & 0 & 0 & 0 & 6 & 4 \\
\hline 11111 & & & 40 & & \\
\hline
\end{tabular}

Notes: For the EQ5D, respondents were asked to describe their health in terms of five dimensions (mobility, self-care, usual activities, pain, and mood), each with three levels of severity ( $\mathrm{I}=$ broadly none, $2=$ some and $3=$ extreme problems).

Table 3 Patient-caregiver comparisons (only significant variables shown)

\begin{tabular}{lll}
\hline & Life satisfaction & Day affect \\
\hline Mean day affect & 0.606 & 0.611 \\
Life satisfaction & & \\
Problems with usual activities & -0.155 & -0.265 \\
Frequency and intensity of thoughts around health & & 0.133 \\
Frequency and intensity of thoughts around family & & -0.191 \\
Having at least one EQ5D dimension at level 3 & -0.039 (not significant) & -0.241 \\
Responder being a caregiver & & \\
\hline
\end{tabular}

Table 4 Intrusive thoughts

\begin{tabular}{lll}
\hline & Patient, \% & Caregiver, \% \\
\hline Thoughts around health & & 15.0 \\
Many times, "happy" rated greater than or equal to "worried" & 15.0 & 67.5 \\
A few times, "happy" rated greater than or equal to "worried" & 52.5 & 15.0 \\
A few times, "worried" rated greater than "happy" & 8.8 \\
Many times, "worried" rated greater than "happy" & 23.8 & 2.5 \\
Thoughts around family & 56.5 \\
Many times, "happy" rated greater than or equal to "worried" & 29.3 \\
A few times, "happy" rated greater than or equal to "worried" & 5.4 & 49.4 \\
A few times, "worried" rated greater than "happy" & 8.7 & 29.1 \\
Many times, "worried" rated greater than "happy" & 3.8 \\
\hline
\end{tabular}

work for Pfizer. Dummy variables were used to indicate some or extreme problems along each EQ5D dimension (mobility, self-care and so on).

The results are shown in Table 5. Interestingly, anxiety/ depression consistently matters to SWB, and pain and mobility consistently do not. Moderate to extreme problems with anxiety/depression were associated with reduction of between $40 \%$ and $60 \%$ on the various SWB measures for patients and caregivers. As shown previously, there were some anomalous results for mobility, with "some problems walking about" sometimes "better" for SWB than no problems at all.

\section{Explaining SWB and TTO}

Table 6 summarizes the main regression results, which included additional background variables to explain each of the main SWB measures (LS and DA) in terms of the EQ5D, intrusive thoughts, and also, innovatively, in terms of the
SWB of the other person in the pair, that is, patient SWB to explain caregiver SWB and vice versa.

Table 6 also shows the determinants of TTO values for patients only. Since the sample is relatively small, only independent variables found to be significant (at the 10\% level) were retained in each regression to limit the statistical noise created by the presence of insignificant explanatory variables.

Anxiety/depression comes through strongly for three of the four models, but does not appear to be related to patient LS, which is better explained by extreme problems with selfcare and usual activities. Patient LS scores did not appear to be significantly influenced by the frequency and intensity of thoughts about health or family. In contrast, more frequent and negative thoughts about health and family were associated with a roughly $25 \%$ lower DA score. Both LS and DA among caregivers were associated with thoughts about health, but not with thoughts about family. 
Table 5 EQ5D “tariff” models

\begin{tabular}{|c|c|c|c|c|c|}
\hline & LS patient & DA patient & LS caregiver & DA caregiver & TTO patient \\
\hline Constant & 86.62 & 3.62 & 88.06 & 3.08 & -0.134 \\
\hline Mobility 2 & -0.022 & 0.024 & -0.089 & 0.119 & 0.109 \\
\hline Mobility 3 & N/A & $\mathrm{N} / \mathrm{A}$ & $\mathrm{N} / \mathrm{A}$ & $\mathrm{N} / \mathrm{A}$ & $\mathrm{N} / \mathrm{A}$ \\
\hline Self-care 2 & -0.157 & -0.129 & -0.084 & $-0.201 *$ & 0.078 \\
\hline Self-care 3 & -0.108 & -0.007 & $\mathrm{~N} / \mathrm{A}$ & $\mathrm{N} / \mathrm{A}$ & -0.013 \\
\hline Usual activities 2 & -0.170 & $-0.182 *$ & -0.190 & -0.169 & -0.052 \\
\hline Usual activities 3 & $-0.347 *$ & $-0.363 *$ & $\mathrm{~N} / \mathrm{A}$ & $\mathrm{N} / \mathrm{A}$ & 0.010 \\
\hline Pain/distress 2 & 0.041 & 0.002 & -0.033 & 0.009 & 0.010 \\
\hline Pain/distress 3 & -0.146 & -0.288 & 0.274 & -0.081 & $-0.450 *$ \\
\hline Anxiety/depression 2 & -0.133 & $-0.235 *$ & $-0.410 *$ & $-0.487 *$ & $-0.167 *$ \\
\hline Anxiety/depression 3 & $-0.44 I *$ & $-0.459 *$ & $-0.464^{*}$ & $-0.64 I *$ & $-0.233^{*}$ \\
\hline Any 3 & $0.427 *$ & 0.347 & -0.184 & -0.378 & 0.050 \\
\hline$R^{2}$ & 0.160 & 0.286 & 0.472 & 0.358 & 0.199 \\
\hline
\end{tabular}

Notes: Significance at $10 \%$ in bold and asterisk. For each parameter, levels of severity are I: broadly none, 2: some, and 3: extreme problems.

Abbreviations: LS, life satisfaction; DA, day affect; TTO, time trade-off; N/A, not available.

Table 6 Summary of regression results (only significant variables shown)

\begin{tabular}{|c|c|c|c|c|c|}
\hline & LS patient & DA Patient & LS caregiver & DA caregiver & TTO patient \\
\hline Self-care 3 & -0.204 & & & & \\
\hline Usual activities 3 & -0.238 & & & & \\
\hline Pain/distress 3 & & & & & -0.204 \\
\hline Anxiety/depression 2 & & -0.192 & -0.232 & -0.323 & \\
\hline Anxiety/depression 3 & & -0.206 & -0.379 & -0.353 & -0.206 \\
\hline Any 3 & -0.347 & & & & \\
\hline Thoughts: health & & -0.219 & 0.242 & -0.326 & \\
\hline Thoughts: family & & -0.236 & & & 0.376 \\
\hline LS patient & & 0.540 & 0.251 & & \\
\hline DA patient & $0.74 I$ & & & & 0.315 \\
\hline LS caregiver & 0.196 & -0.169 & & 0.387 & \\
\hline DA caregiver & & & 0.392 & & 0.205 \\
\hline Cancer stage IV & -0.061 & & -0.144 & & \\
\hline Cancer in remission & -0.152 & 0.154 & & 0.182 & \\
\hline$R^{2}$ & 0.582 & 0.684 & 0.625 & 0.730 & 0.460 \\
\hline
\end{tabular}

Abbreviations: LS, life satisfaction; DA, Day affect; TTO, time trade-off.

Being a stage IV cancer patient, a group comprising over half the study's patient population, was found to have a negative effect on the LS of both patients and caregivers, with an associated reduction of $6 \%$ and $14 \%$, respectively. Interestingly, patient LS was found to be negatively affected by cancer going into remission, with an associated $15 \%$ reduction in LS. Being in remission was good for patient DA (and caregiver DA too), with increases of $15 \%$ and $18 \%$, respectively.

Table 6 also shows the significant association found between LS and DA within both patients and caregivers, and also some relationship across the groups. Patient LS was significantly affected by caregiver LS scores. Caregiver LS was associated with patient DA scores, although in the unexpected direction, with higher caregiver LS being associated with lower patient DA.
Age, gender, marital status, employment and education data were not found to be significant in predicting SWB scores.

Unsurprisingly, TTO values were explained by extreme pain/discomfort and by extreme anxiety/depression. More innovatively, TTO values were not explained by thoughts about health, but by thoughts about family - the better those thoughts were, the lesser the willingness to give up life years to avoid current health problems. A further novel finding is that better DA of both patient and caregiver was associated with less willingness to give up life years.

\section{Conclusion and recommendations}

In principle, there is no doubt that QALYs are a powerful tool for allocating health care resources. In practice, there are problems with how the Q in the QALY is generated, that is, 
with how health is described and valued. Descriptive systems, such as the EQ5D, are not really designed to pick up the impact of conditions on non-patient populations such as caregivers.

This is one of the few studies that have elicited SWB ratings (and TTO values) from a clinical population. The study focuses on patients with colorectal cancer and their caregivers. It is best described as a pilot study involving 99 patients and 88 caregivers, and our data allow us to directly compare the SWB of these two groups (objective 3). The first thing to note is that caregivers are just as anxious or depressed as patients and, at face value, this highlights the potential importance of support being available to families of cancer patients as well as to the patients themselves.

The LS scores for patients and caregivers were nearly identical, but patients had significantly higher DA. These results potentially further demonstrate that caregivers are significantly impacted by cancer and are potential beneficiaries of treatment. Although patients reported worse health on the EQ5D (with only $16 \%$ in 11111 as compared to $40 \%$ of caregivers, with "11111" indicating "broadly no" problems in the five EQ5D dimensions), the proportions reporting problems with anxiety/ depression are the same across the two groups and, from this and other work, it is strongly suggested that anxiety/depression does the "heavy lifting" in SWB. Both caregiver LS and caregiver DA are strongly associated with anxiety/depression.

The results in Table 5 confirm previous analyses, which basically show that mental health is the most important dimension for SWB and pain/discomfort is additionally important for TTO. The sample sizes are small, and we should be cautious about making inferences about what does not affect SWB, but very few dimensions and levels of the EQ5D are significant in explaining LS and DA. We might need to face up to the fact that limitations to mobility, for example, really do not significantly affect our SWB.

We have discussed the importance of mental health as the major determinant of SWB in a previous work supported by Pfizer. This highlights that treatments that bring about reduction in anxiety or depression (or other psychiatric symptoms) are equally important. From studies in the general population, we know that the importance of mental health was strongest for LS, but here the impact is broadly comparable across both the main measures of SWB.

In exploring the relationships between SWB and the background characteristics of the respondents (objective 5), the results for patient LS suggest some interesting findings more generally. In particular, being in remission is associated with lower LS. This seems counterintuitive, since remission involves the prospect of better health and a longer life. It could be, however, that active cancer allows people to make sense of their lives and find meaning in the face of adversity. On the other hand, being in remission is good for the DA of both patients and caregivers. Cancer in stage IV was associated with a significant reduction in caregiver LS and cancer being in remission was good for the DA of caregivers.

It is also worth noting the role thoughts about health and family play in explaining differences between the two main SWB measures. Such thoughts do not seem to affect LS as much as they affect DA (especially for patients). This might suggest that DA might be failing to capture some important effects on SWB that result from mind wanderings. ${ }^{16}$

By eliciting TTO values from patients, we were able to explore the impact of SWB on these values, as well as to look for effects of the usual suspects relating to the EQ5D (objective 6). The pain and mood dimensions of the EQ5D affect TTO values. While thoughts about health do not appear to affect TTO values, ${ }^{19}$ those who have more thoughts about their family are less willing to trade-off life years, and this makes good sense. Better DA of both patient and caregiver also results in greater reluctance to give up life years.

It is noteworthy that none of the "standard" background variables (age, gender, education and so on) had much effect on SWB and TTO. Of course, with larger sample sizes, some of these effects may have been significant, so we should be cautious about claiming such factors do not matter. Indeed, the burgeoning literature on SWB suggests that many of these background variables are indeed important determinants of SWB ${ }^{20}$ Perhaps another important factor in patients' QoL that was not included in our study is the use of complementary and alternative medicines. Given the increasingly evolving evidence on the impact of complementary medicine in cancer patients' QoL, it is worth considering adding questions on their use in future questionnaires. ${ }^{21}$

This pilot study has provided some useful insights into the SWB of cancer patients and their caregivers, and we now need larger, longitudinal studies that more closely monitor the impact of cancer on patients and caregivers. Such research would begin to shed light on how different cancer and health states causally affect SWB and how SWB causally affects cancer and health states. There is some good evidence on the causal effects that SWB has on a range of outcomes, including health, ${ }^{22}$ and so it may be that effective interventions in health care might be those that seek to improve SWB.

Some of the results from this pilot study have been surprising. Firstly, the finding that LS is lower for patients who are in remission is a particularly noteworthy finding that requires further investigation into the degree to which it reflects a "genuine effect" and the degree to which it reflects some kind of cognitive dissonance on the part of patients. 
While anxiety/depression emerged as an important determinant of SWB, its greater effect on DA relative to LS in this study is slightly at odds with what we found in the previous work with the general population; further research should explore the reason for these differences.

The fact that pain matters greatly in TTO preferences but hardly shows up at all in SWB is intriguing, and we need to examine the robustness of this difference more fully. As shown before, pain relief would be less cost-effective using an SWB-based approach. ${ }^{23}$

This study provides insights on the determinants of well-being in cancer patients and their caregivers and the real-world benefits of treatment. Our future work will build on the pilot study reported here.

\section{Acknowledgments}

We sincerely thank Paul Dolan, Professor of Behavioral Sciences (London School of Economics, London), for his support and for designing the patient/caregiver questionnaire and allowing us to use it as part of this study. We would also like to thank James Piercy (Adelphi Real World) and Warren Cowell (Pfizer) for their contributions to this work.

This work was financially supported by Pfizer. Adelphi Real World, a third-party research organization, was involved in collecting, processing, handling and storing the information on behalf of Pfizer. The study was conducted by the Clinical Trials Unit at the Beatson, West of Scotland Cancer Center, Glasgow, UK.

\section{Author contributions}

All named authors have read the manuscript and have agreed to submit the paper in its present form. All authors contributed toward data analysis, drafting and revising the paper and agree to be accountable for all aspects of the work.

\section{Disclosure}

Julie Ann Bridge was employed by Pfizer Ltd, UK at the time of the study. The authors report no other conflicts of interest in this work.

\section{References}

1. Maisey NR, Norman A, Watson M, Allen MJ, Hill ME, Cunningham D. Baseline quality of life predicts survival in patients with advanced colorectal cancer. Eur J Cancer. 2002;38:1351-1357
2. Montazeri A. Quality of life data as prognostic indicators of survival in cancer patients: an overview of the literature from 1982 to 2008. Health and Qual of Life Outcomes 2009,7:102

3. Quinten C, Coens C, Mauer M, et al. Baseline quality of life as a prognostic indicator of survival: a meta-analysis of individual patient data from EORTC clinical trials. The Lancet Oncol 2009;10(9): $865-871$

4. Vera-Badillo FE, Shapiro R, Ocana A, Amir E., Tannock IF. Bias in reporting of end-points of efficacy and toxicity in randomized clinical trials for women with breast cancer. Ann Oncol 2013; 24(5):1238-1244.

5. EuroQol Group. EuroQol-a new facility for the measurement of healthrelated quality of life. Health Policy, 1990;16: 199-208.

6. Brazier J, Roberts J, Deverill M. The estimation of a preferencebased measure of health from the SF-36. J Health Econ. 2002;21(2): 271-292.

7. NICE. Guide to the methods of technology appraisal. National Institute for Clinical Excellence 2008. Available from: https://www.nice.org.uk/ process/pmg9/chapter/foreword.

8. Graham C. Happiness and health: lessons-and questions-for public policy. Health Aff (Millwood). 2008;27(1):72-87.

9. Pressman SD, Cohen S. Does positive affect influence health? Psychol Bull. 2005;131(6):925-971.

10. Barak Y. The immune system and happiness. Autoimmun Rev. 2006; 5(8):523-527.

11. Nijboer C, Triemstra M, Tempelaar R, Sanderman R, van den Bos GA. Determinants of caregiving experiences and mental health of partners of cancer patients. Cancer. 1999;86(4):577-588.

12. Kornblith AB, Herr HW, Ofman US, Scher HI, Holland JC. Quality of life of patients with prostate cancer and their spouses. The value of a data base in clinical care. Cancer. 1994;73(11):2791-2802.

13. Harding R, Higginson IJ, Donaldson N. The relationship between patient characteristics and caregiver psychological status in home palliative cancer care. Support Care Cancer. 2003;11(10):638-643.

14. Dolan P, Fujiwara D, Peasgood T. The impact of caregiver burden. Report to the Department of Health; 2010.

15. Dolan P, Lee H, Peasgood T. Losing sight of the wood for the trees: Some issues in describing and valuing health and another possible approach. Pharmaceconomics. 2012 30(11):1035-1049.

16. Smallwood J, Schooler JW. The restless mind. Psychol Bull. 2006; 132(6):946-958.

17. Watkins ER. Constructive and unconstructive repetitive thought. Psychol Bull. 2008;134(2):163-206.

18. Killingsworth MA, Gilbert DT. A wandering mind is an unhappy mind. Science. 2010;330(6006):932.

19. Dolan P. Thinking about it: thoughts about health and valuing QALYs. Health Econ. 2011;20(12):1407-1416.

20. Dolan P, Peasgood T, White M. Do we really know what makes us happy? A review of the economic literature on the factors associated with subjective well-being. J Econ Psychol. 2008;29(1):94-122.

21. Frass M, Friehs H, Thallinger C, et al. Influence of adjunctive classical homeopathy on global health status and subjective wellbeing in cancer patients - A pragmatic randomized controlled trial. Complement Ther Med. 2015;23(3):309-317.

22. Lyubomirsky S, King L, Diener E. The benefits of frequent positive affect: does happiness lead to success? Psychol Bull. 2005;131(6): 803-855.

23. Dolan P. Using Happiness to Value Health. Office of Health Economics. 2011. Available from: http://pauldolan.co.uk/wp-content/ uploads/2012/01/Using_Happiness_to_value_health.pdf. 


\section{Publish your work in this journal}

Patient Related Outcome Measures is an international, peer-reviewed, open access journal focusing on treatment outcomes specifically relevant to patients. All aspects of patient care are addressed within the journal and practitioners from all disciplines are invited to submit their work as well as healthcare researchers and patient support groups.
The journal is included in PubMed. The manuscript management system is completely online and includes a very quick and fair peer-review system. Visit http://www.dovepress.com/testimonials.php to read real quotes from published authors.

Submit your manuscript here: http://www.dovepress.com/patient-related-outcome-measures-journal 